\title{
Long noncoding RNA NEAT1, regulated by LIN28B, promotes cell proliferation and migration through sponging miR-506 in high-grade serous ovarian cancer
}

Wu Yong ${ }^{1,2}$, Deng Yu ${ }^{2,3}$, Zhu Jun ${ }^{1,2}$, Duan Yachen ${ }^{1,2}$, Weng Weiwei ${ }^{2,3}$, Xu Midie $^{2,3}$, Ju Xingzhu ${ }^{1,2}$ and Wu Xiaohua ${ }^{1,2}$

\begin{abstract}
The aberrant expression of long noncoding RNAs (IncRNAs) has been reported frequently in specific cancers, including high-grade serous ovarian cancer (HGSOC). The purpose of the present study was to explore the clinical significance and underlying mechanisms of a significantly dysregulated IncRNA (NEAT1) in HGSOC. Our results showed that elevated NEAT1 expression in human HGSOC specimens correlated with a poor prognosis. Functional experiments demonstrated that knockdown of NEAT1 significantly prohibited ovarian cancer cell proliferation and invasion in vitro and restrained tumor growth in vivo. LIN28B was identified by bioinformatics analysis along with experimental evidence as a direct actor that enhanced NEAT1 stability. A rescue functional assay confirmed that the LIN28B/NEAT1 axis contributed to oncogenic functions in ovarian cancer cells. Moreover, gene expression profile data and dual luciferase reporter assay results demonstrated that NEAT1 functioned as a competing endogenous RNA (ceRNA) for miR-506 to promote cell proliferation and migration. Taken together, our results showed that NEAT1, stabilized by LIN28B, promoted HGSOC progression by sponging miR-506. Thus, NEAT1 can be regarded as a vital diagnostic biomarker for HGSOC and a therapeutic target.
\end{abstract}

\section{Introduction}

Epithelial ovarian cancer (EOC) is the most lethal gynecological cancer and a common cause of cancerrelated death in women worldwide ${ }^{1,2}$. Despite aggressive frontline treatments with surgery and targeted chemotherapy, most patients relapse and die from their disease $^{2}$. High-grade serous ovarian carcinoma (HGSOC) accounts for $60-80 \%$ of the women diagnosed with EOC, and most deaths related to EOC are associated with this subtype $^{3}$. Therefore, understanding the pathophysiological mechanisms contributing to HGSOC is of paramount

\footnotetext{
Correspondence: Wu Xiaohua (wu.xh@fudan.edu.cn)

'Department of Gynecologic Oncology, Fudan University Shanghai Cancer

Center, Shanghai, China

${ }^{2}$ Department of Oncology, Shanghai Medical College, Fudan University,

Shanghai, China

Full list of author information is available at the end of the article.

Edited by G. Calin
}

importance for the development of new diagnostic techniques and treatment strategies and the improvement of the overall prognosis of $\mathrm{OC}$ patients.

Long noncoding RNAs (lncRNAs), which are a newly discovered class of noncoding RNA (ncRNA) greater than 200 nucleotides in length, have been increasingly reported in a variety of cancer types, suggesting an important role of lncRNAs in human diseases, especially cancer ${ }^{4,5}$. Many studies have demonstrated the diverse cellular functions of lncRNAs, including cell proliferation, cell differentiation, cell apoptosis, and carcinogenesis ${ }^{5,6}$. NEAT1 is an abundant intranuclear lncRNA that contains two transcripts, NEAT1_1 (3.7 kb) and NEAT1_2 (23 kb); the latter transcript is a core component of paraspeckles, which are major complexes involved in RNA nuclear retention that participate in precursor RNA splicing ${ }^{7-10}$. Previous studies have suggested that NEAT1 is an

\section{(c) The Author(s) 2018}

(c) (i) Open Access This article is licensed under a Creative Commons Attribution 4.0 International License, which permits use, sharing, adaptation, distribution and reproduction c. in any medium or format, as long as you give appropriate credit to the original author(s) and the source, provide a link to the Creative Commons license, and indicate if changes were made. The images or other third party material in this article are included in the article's Creative Commons license, unless indicated otherwise in a credit line to the material. If material is not included in the article's Creative Commons license and your intended use is not permitted by statutory regulation or exceeds the permitted use, you will need to obtain permission directly from the copyright holder. To view a copy of this license, visit http://creativecommons.org/licenses/by/4.0/. 


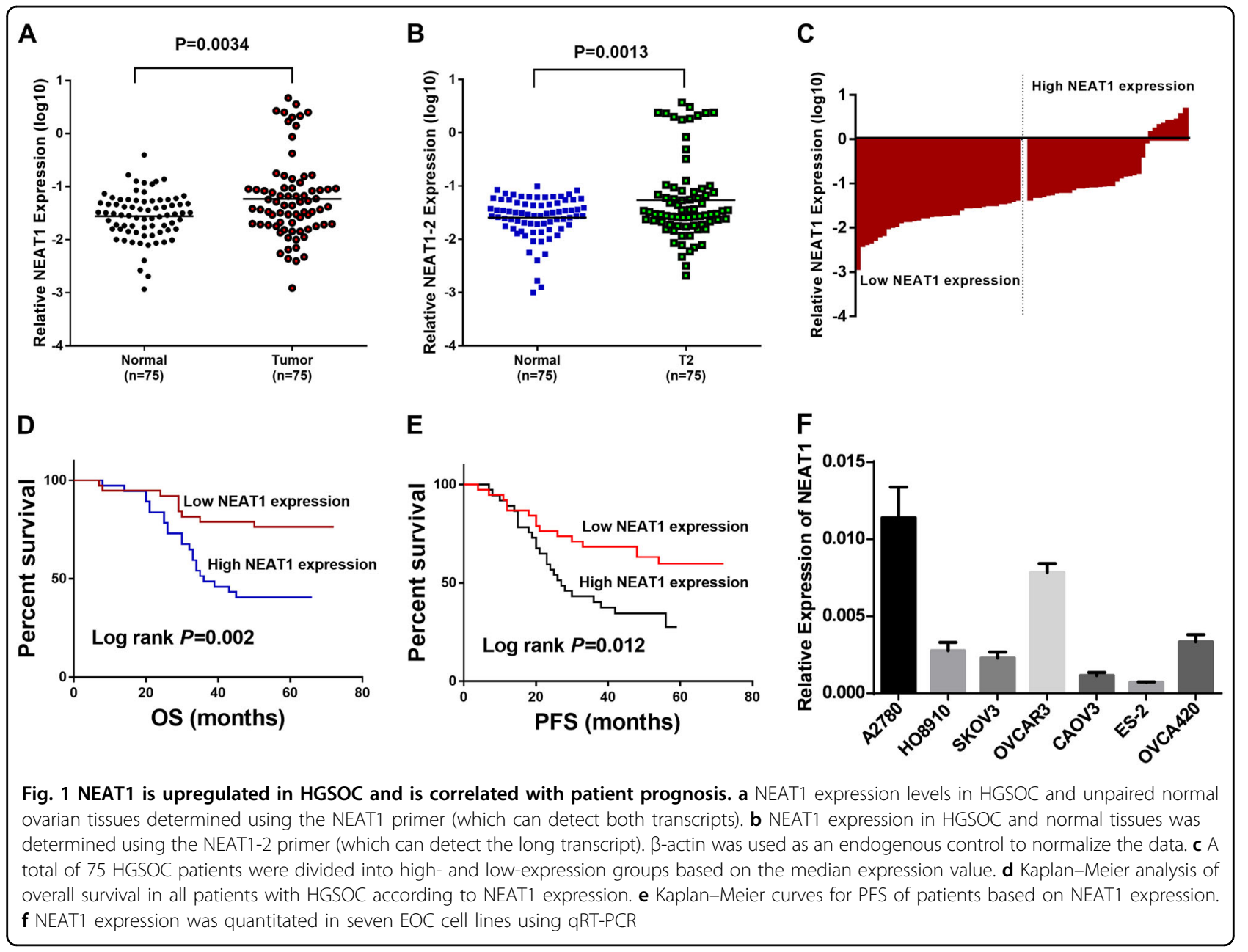

oncogene in various cancers, including lung cancer ${ }^{11}$, hepatocellular cancer ${ }^{12}$, prostate cancer $^{13}$, colorectal cancer ${ }^{14}$, and nasopharyngeal carcinoma ${ }^{15,16}$. Although some studies have revealed that NEAT1 may exhibit malignant biological behaviors in $\mathrm{EOC}^{17}$, the detailed mechanisms and functions of NEAT1 in HGSOC have not been clearly elucidated.

Recently, growing knowledge of RNA-binding protein (RBP) targets has directed attention towards ncRNAs, including RNAs involved in translation machinery and its regulation (rRNAs, tRNAs, siRNAs, and miRNAs) as well as the large and heterogeneous class of lncRNAs ${ }^{18,19}$. However, only a small number of lncRNAs have been functionally well characterized to date ${ }^{20,21}$. A few reports have noted that NEAT1 can bind RBPs, such as NONO and PSF $^{22}$. However, relationships between NEAT1 and other RBPs have rarely been reported.

In this study, we found that NEAT1 was overexpressed in HGSOC tissues and that this lncRNA promoted cell proliferation, migration, and invasiveness as well as tumor growth in vivo. Furthermore, mechanistic investigations showed that the upregulation of NEAT1 in HGSOC was mediated by the RBP LIN28B, which bound to and stabilized NEAT1. By determining the downstream effects of NEAT1, our results suggested that the LIN28B/NEAT1 axis might confer an oncogenic function via sponging miR-506. These findings provide new insights into the molecular functions of NEAT1 and shed new light on the treatment of HGSOC.

\section{Results}

NEAT1 is upregulated in HGSOC and correlates with poor outcomes

Considering that NEAT1 has two transcripts that share the same $5^{\prime}$ end but are processed alternatively at the $3^{\prime}$ terminus $^{22}$, it was of interest to determine whether one transcript plays a major oncogenic role in HGSOC or the two transcripts have similar roles. To do so, we silenced NEAT1 via an siRNA targeting both NEAT1 transcripts or an siRNA targeting NEAT1-2 only. The two siRNAs resulted in the nearly identical arrest of ovarian cancer cell proliferation and migration (Supplementary 
Figure S1A, B, C), which suggested that targeting only NEAT1-2, which was recognized as the predominant isoform for the function of NEAT1 in the paraspeckle, did not have a stronger oncogenic effect. Then, we designed two primers named NEAT1 (which can detect both transcripts) and NEAT1-2 (which can detect the long transcript) to assess their expression levels in HGSOC tissues. The qPCR analysis showed that both total NEAT1 and NEAT1-2 were expressed at significantly higher levels in HGSOC tissues than in normal ovarian tissues (Fig. 1a, b; $P=0.0034, P=0.0013)$. Thus, we used the NEAT1 primer in our subsequent experiments unless otherwise specified. Based on the above results, we speculated that the two transcripts share the major oncogenic role in HGSOC. Therefore, siRNAs targeting the common sequences of both transcripts were designed for the subsequent functional experiments.

For the clinicopathological correlation analysis, 75 HGSOC patients were divided into high- and lowexpression groups based on the median expression value (Fig. 1c). As shown in Table 1, high NEAT1 expression was closely associated with tumor size $(P=0.006)$. Overall survival (OS) and progression-free survival (PFS) curves were plotted according to the NEAT1 expression levels using the Kaplan-Meier method and log-rank test. As shown in Fig. 1d, e, high NEAT1 expression was significantly correlated with both shorter OS $(P=0.002)$ and shorter PFS $(P=0.012)$. The univariate analysis revealed that the relative NEAT1 expression level $(P=0.004 ; P=$ 0.015), International Federation of Gynecology and Obstetrics (FIGO) stage $(P=0.016 ; P=0.036)$, and vascular invasion $(P=0.038 ; P=0.028)$ were prognostic indicators of OS (Table 2) and PFS (Table 3). Moreover, the multivariate Cox regression analysis showed that FIGO stage $(P=0.011 ; P=0.006)$, vascular invasion $(P=$ $0.022 ; P=0.011)$, and high NEAT1 expression $(P=0.007$; $P=0.026$ ) were independent risk factors of OS (Table 2) and PFS (Table 3). These results suggest that NEAT1 is a potential independent prognostic factor for HGSOC. Furthermore, we determined the NEAT1 expression in the EOC cell lines OVCAR3 and A2780, which were selected for subsequent knockdown experiments because of their high relative expression (Fig. 1f).

\section{Attenuation of NEAT1 expression inhibits cell proliferation and invasion}

To verify that NEAT1 expression was positively associated with HGSOC progression, we first employed four siRNA oligonucleotides to test the knockdown efficiency for this IncRNA in OVCAR3 and A2780 cells. The qPCR results (Fig. 2a) showed that NEAT1-SI2 and NEAT-SI4 significantly reduced both total NEAT1 and NEAT1-2 endogenous expression. Therefore, we chose these two siRNAs to perform the functional experiments. We
Table 1 The correlation between NEAT1 and clinicopathological parameters

\begin{tabular}{|c|c|c|c|c|c|}
\hline \multirow[t]{2}{*}{ Variables } & \multirow[t]{2}{*}{$n(\%)$} & \multicolumn{2}{|c|}{$\begin{array}{l}\text { Expression } \\
\text { of NEAT1 }\end{array}$} & \multirow[t]{2}{*}{$x^{2}$} & \multirow[t]{2}{*}{$P$ value } \\
\hline & & Low & High & & \\
\hline Age (years) & & & & 2.259 & 0.133 \\
\hline$\leq 55$ & $38(50.6)$ & 23 & 15 & & \\
\hline$>55$ & $37(49.4)$ & 15 & 22 & & \\
\hline Size $(\mathrm{cm})$ & & & & 7.469 & 0.006 \\
\hline$\leq 5$ & $27(36.0)$ & 13 & 14 & & \\
\hline$>5$ & $48(64.0)$ & 25 & 23 & & \\
\hline FIGO stage & & & & 0.108 & 0.742 \\
\hline$|-| \mid$ & $21(28.0)$ & 10 & 11 & & \\
\hline||$|-| V$ & $54(72.0)$ & 28 & 26 & & \\
\hline Poor histologic differentiation & & & & 0.015 & 0.902 \\
\hline Yes & $35(46.7)$ & 18 & 17 & & \\
\hline No & $40(53.3)$ & 20 & 20 & & \\
\hline Vascular invasion & & & & 1.382 & 0.240 \\
\hline Yes & $23(30.7)$ & 14 & 9 & & \\
\hline No & $52(69.3)$ & 24 & 28 & & \\
\hline Lymphatic metastasis & & & & 3.316 & 0.069 \\
\hline Yes & $28(37.3)$ & 18 & 10 & & \\
\hline No & $47(62.7)$ & 20 & 27 & & \\
\hline Distant metastasis & & & & 1.139 & 0.286 \\
\hline Yes & $16(21.3)$ & 10 & 6 & & \\
\hline No & 59 (78.7) & 28 & 31 & & \\
\hline
\end{tabular}

utilized the CCK8 and colony formation assays to elucidate the potential effect of NEAT1 on cell proliferation. The CCK8 assay showed that cell viability was significantly restrained after NEAT1 downregulation $(P<$ 0.05; Fig. 2b). Consistently, the colony formation assays showed that knockdown of NEAT1 led to the formation of fewer colonies in both the OVCAR3 and A2780 cells than in the control cells without NEAT1 knockdown $(P<$ 0.05; Fig. 2c). These data suggest that knockdown of NEAT1 represses tumor cell proliferation.

Because cell invasion is an important aspect of cancer progression that involves the migration of tumor cells into contiguous tissues and the dissolution of extracellular matrix proteins, we evaluated the effects of NEAT1 on cell migration and invasion. The Transwell assay results shown in Fig. 2d demonstrated that transfection of the siRNAs targeting NEAT1 impeded the migratory ability of the cells $(P<0.05)$. In addition, the wound assay showed that the ovarian cancer cells transfected with the siRNAs 
Table 2 Univariate and multivariate analysis of clinicopathological factors for overall survival

\begin{tabular}{|c|c|c|c|c|c|c|}
\hline \multirow[t]{2}{*}{ Variables } & \multicolumn{3}{|c|}{ Univariate analysis } & \multicolumn{3}{|c|}{ Multivariate analysis } \\
\hline & HR & $95 \% \mathrm{Cl}$ & $P$ value & HR & $95 \% \mathrm{Cl}$ & $P$ value \\
\hline NEAT1 expression & 3.315 & $1.439-6.828$ & $0.004^{*}$ & 2.915 & $1.333-6.377$ & $0.007^{*}$ \\
\hline FIGO stage $(|-|||||-,\mid \mathrm{V})$ & 3.088 & $1.079-8.833$ & $0.016^{*}$ & 3.953 & $1.369-11.413$ & $0.011^{*}$ \\
\hline Vascular invasion (Yes, No) & 0.362 & $0.139-0.943$ & $0.038^{*}$ & 0.322 & $0.122-0.849$ & $0.022^{*}$ \\
\hline Lymphatic metastasis & 0.586 & $0.270-1.273$ & 0.177 & & & \\
\hline Age $(\leq 55,>55)$ & 1.014 & $0.501-2.053$ & 0.969 & & & \\
\hline Size $(\leq 5 \mathrm{~cm},>5 \mathrm{~cm})$ & 0.569 & $0.280-1.155$ & 0.119 & & & \\
\hline Poor histologic differentiation (Yes, No) & 0.911 & $0.450-1.843$ & 0.796 & & & \\
\hline Distant metastasis (Yes, No) & 1.070 & $0.461-2.483$ & 0.875 & & & \\
\hline
\end{tabular}

$H R$ hazard ratio

${ }^{*} P<0.05$

Table 3 Univariate and multivariate analysis of clinicopathological factors for progression-free survival

\begin{tabular}{|c|c|c|c|c|c|c|}
\hline \multirow[t]{2}{*}{ Variables } & \multicolumn{3}{|c|}{ Univariate analysis } & \multicolumn{3}{|c|}{ Multivariate analysis } \\
\hline & HR & $95 \% \mathrm{Cl}$ & $P$ value & HR & $95 \% \mathrm{Cl}$ & $P$ value \\
\hline NEAT1 expression & 2.234 & $1.170-4.266$ & $0.015^{*}$ & 2.096 & $1.092-4.023$ & $0.026^{*}$ \\
\hline FIGO stage (I-II, III-IV) & 2.399 & $1.059-5.432$ & $0.036^{*}$ & 3.194 & $1.390-7.337$ & $0.006^{*}$ \\
\hline Vascular invasion (Yes, No) & 0.418 & $0.192-0.911$ & $0.028^{*}$ & 0.356 & $0.160-0.789$ & $0.011^{*}$ \\
\hline Lymphatic metastasis & 0.660 & $0.340-1.282$ & 0.220 & & & \\
\hline Age $(\leq 55,>55)$ & 0.840 & $0.452-1.564$ & 0.583 & & & \\
\hline Size $(\leq 5 \mathrm{~cm},>5 \mathrm{~cm})$ & 0.844 & $0.445-1.603$ & 0.605 & & & \\
\hline Poor histologic differentiation (Yes, No) & 1.167 & $0.623-2.185$ & 0.630 & & & \\
\hline Distant metastasis (Yes, No) & 1.119 & $0.533-2.352$ & 0.766 & & & \\
\hline
\end{tabular}

$H R$ hazard ratio

${ }^{*} P<0.05$

had lower migration capacities than did the control cells (Fig. 2e). Next, we evaluated the expression of epithelial-mesenchymal transition (EMT)-associated proteins and matrix metalloproteinases (MMPs). Western blotting analysis revealed that the epithelial marker gene E-cadherin was significantly increased in the NEAT1knockdown group relative to the control group (Fig. 2f), whereas the mesenchymal marker $\mathrm{N}$-cadherin was significantly decreased (Fig. 2f). In addition, MMP9 and MMP2 were downregulated in the NEAT1-knockdown group (Fig. 2f). These findings suggested that NEAT1 influenced the migration and invasion of EOC cells.

\section{NEAT1 interacts with the RBP LIN28B in tumor cells}

To explore the molecular mechanism underlying the oncogenic activity of NEAT1 in ovarian carcinogenesis, StarBase V2.0 (http://starbase.sysu.edu.cn/mirLncRNA. php) and RNA-Protein Interaction Prediction (http:// pridb.gdcb.iastate.edu/RPISeq/) were used to predict the interaction probabilities of NEAT1 and RBPs. The bioinformatics analysis revealed that NEAT1 could potentially bind to LIN28B. To investigate the potential interaction between the IncRNA NEAT1 and LIN28B, RNA pulldown assays (Fig. 3a) were conducted with the A2780 cell line. In view of the length of NEAT1, we first explored the possible binding sites through CLIP data using the ENCODE database (https://www.encodeproject. org/experiments/ENCSR861GYE/); detailed binding information is provided in Supplementary Table 1. Then, two credible binding sites were chosen to design RNA probes and corresponding mutant probes (Fig. 3b). Subsequently, we confirmed the interaction between NEAT1 and LIN28B through RNA pulldown and Western blotting assays (Fig. 3c). We also performed RNA 


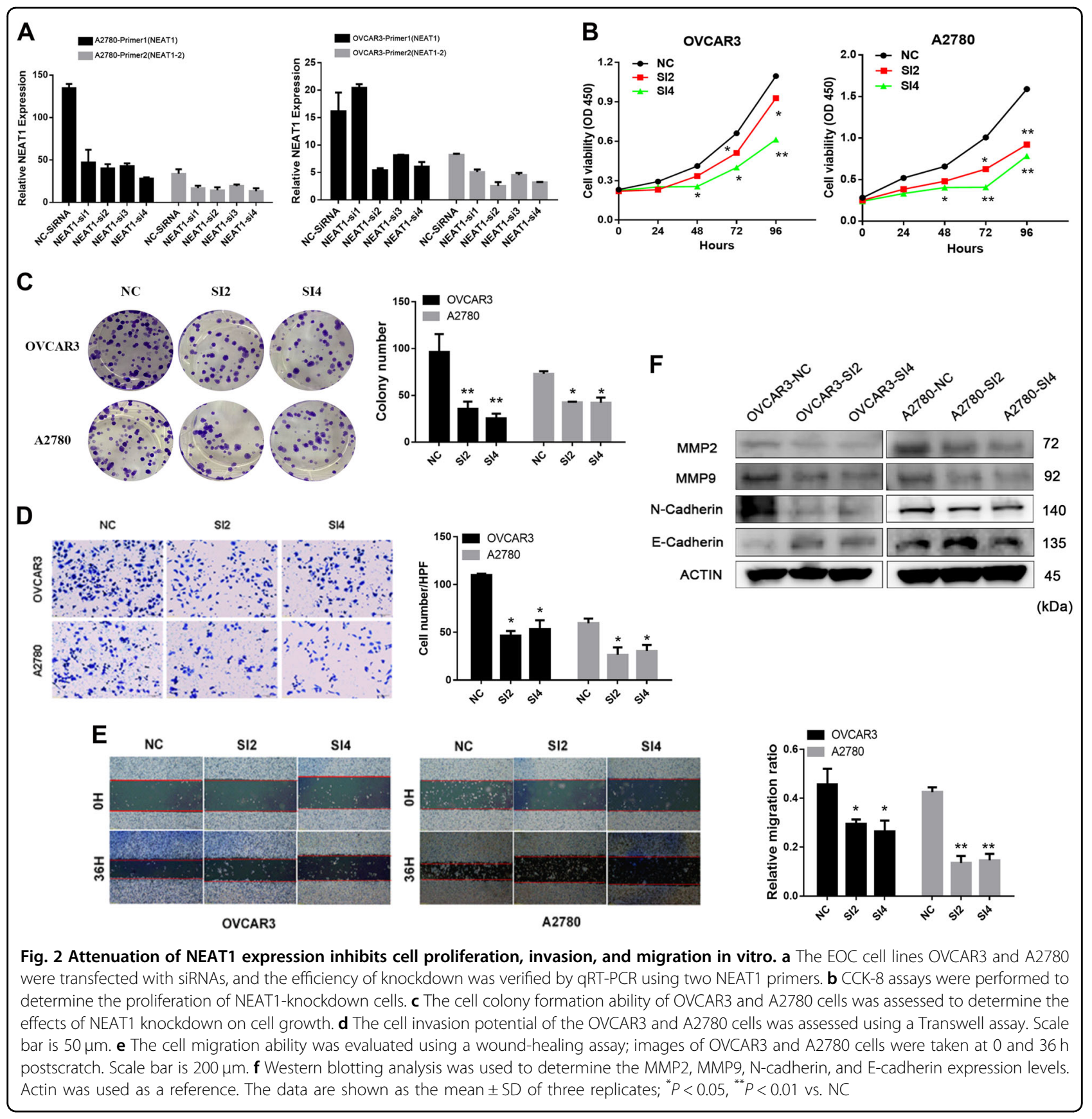

immunoprecipitation followed by detection of NEAT1 enrichment using qPCR and found that NEAT1 was enriched in the LIN28B samples relative to the IgG group (Fig. 3d). As expected, obvious enrichment of H19 was observed in the LIN28B group (Fig. 3d), as has been reported previously ${ }^{23}$. In contrast, LINC00152, which was regarded as the negative control group, could not bind LIN28B (Fig. 3d). Notably, NEAT1-2 has a weak binding capacity with LIN28B. We suspect that these two transcripts may have different secondary structures; the long isoform mainly binds RBPs, such as NONO and $\mathrm{PSF}^{22}$, which may influence the interaction between NEAT1-2 and LIN28B.

\section{LIN28B enhances the stability of NEAT1 in EOC cells}

Given that NEAT1 interacted with LIN28B in EOC cells, we characterized the molecular consequences of these associations. Interestingly, NEAT1 had no effect on the LIN28B protein levels or mRNA levels (Fig. 4a), prompting us to evaluate whether NEAT1 could be regulated by LIN28B in EOC cells. We evaluated the efficiency of overexpression or knockdown of LIN28B 


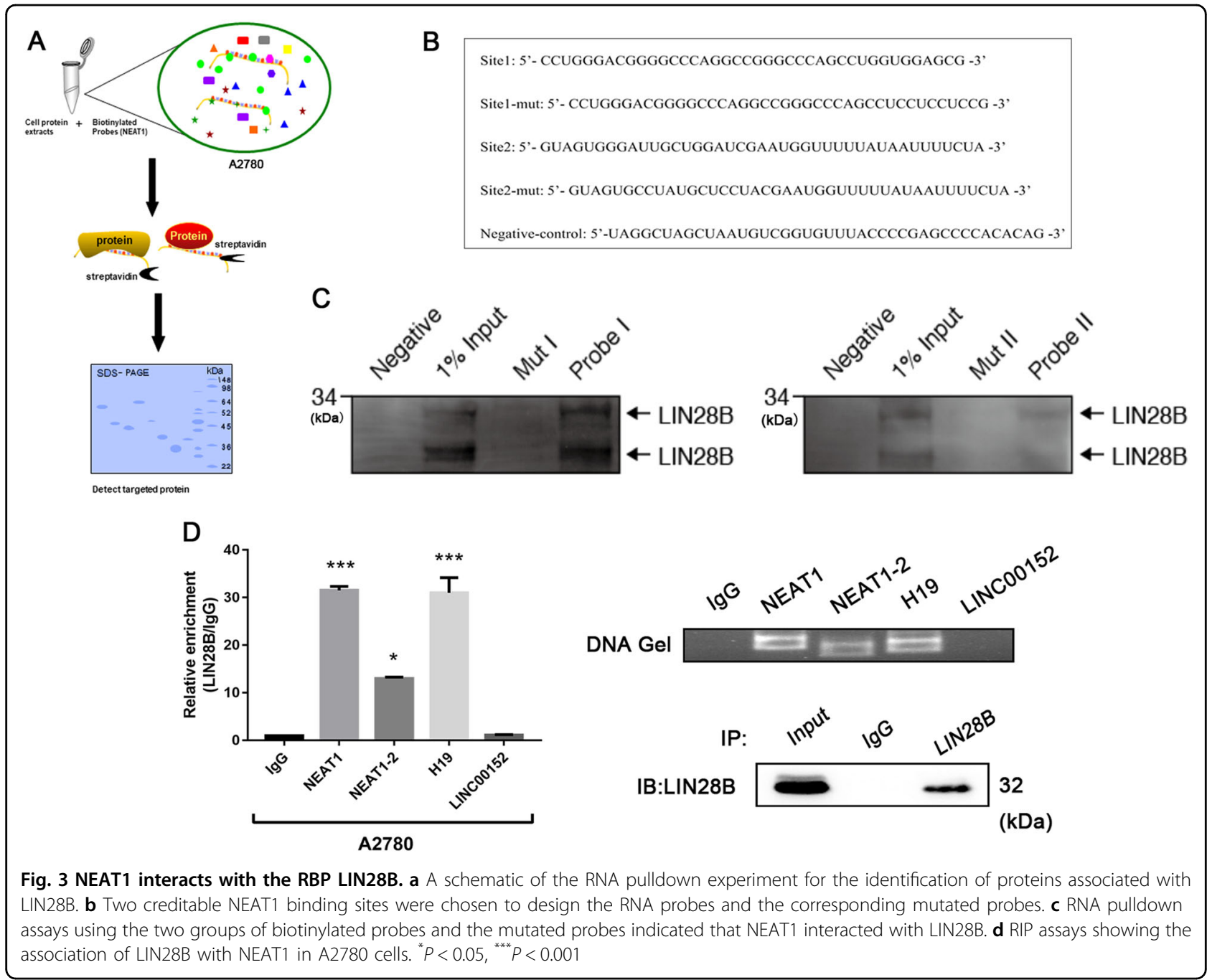

(PCDH: empty control; Fig. 4b, c). Overexpression of LIN28B enhanced the NEAT1 RNA levels, whereas silencing LIN28B decreased the NEAT1 RNA levels in A2780 cells (Fig. 4d, e). Moreover, actinomycin D, which effectively inhibits de novo RNA synthesis, was used to explore the stability of NEAT1. Overexpression of LIN28B resulted in increases in the NEAT1 half-life and RNA level (Fig. 4f), revealing that LIN28B specifically regulated NEAT1 stability in the EOC cells.

\section{NEAT1 is a functional downstream mediator of LIN28B}

Considering the effect of LIN28B on NEAT1 stability, we hypothesized that the biological effects of NEAT1 may be partially regulated by LIN28B. We conducted in vitro experiments to investigate this hypothesis in ovarian cancer cells. The CCK- 8 and colony formation assay results showed that LIN28B overexpression promoted OVCAR3 and A2780 cell proliferation, which was impaired by simultaneous knockdown of NEAT1 (Fig. 5a, b). The cell-wounding and Transwell assays also showed that NEAT1 knockdown partially attenuated the effects of LIN28B overexpression in ovarian cancer cells (Fig. 5c, d). Our results indicated that NEAT1 promoted ovarian cancer cell proliferation and metastasis partly in a LIN28B-regulated manner.

Considering the interaction between LIN28B and NEAT1 and the effect of LIN28B on NEAT1, we examined the LIN28B mRNA levels in HGSOC patients. Quantitative real-time PCR (qRT-PCR) revealed that LIN28B levels were much higher in HGSOC tissues than in normal ovarian tissues (Fig. 5e), and the survival analysis showed that high LIN28B expression was correlated with shorter OS $(P=0.0255$, Fig. $5 \mathrm{~g})$ but had no significant correlation with PFS $(P=0.0794$, Supplementary Figure S2). Moreover, Spearman's correlation analysis suggested a significant positive correlation between LIN28B and each of total NEAT1 and NEAT1-2 in HGSOC tissues $(r=0.5182$ and $0.443, P<0.001$, Fig. $5 f)$, which further confirmed the relationship between LIN28B and NEAT1. 


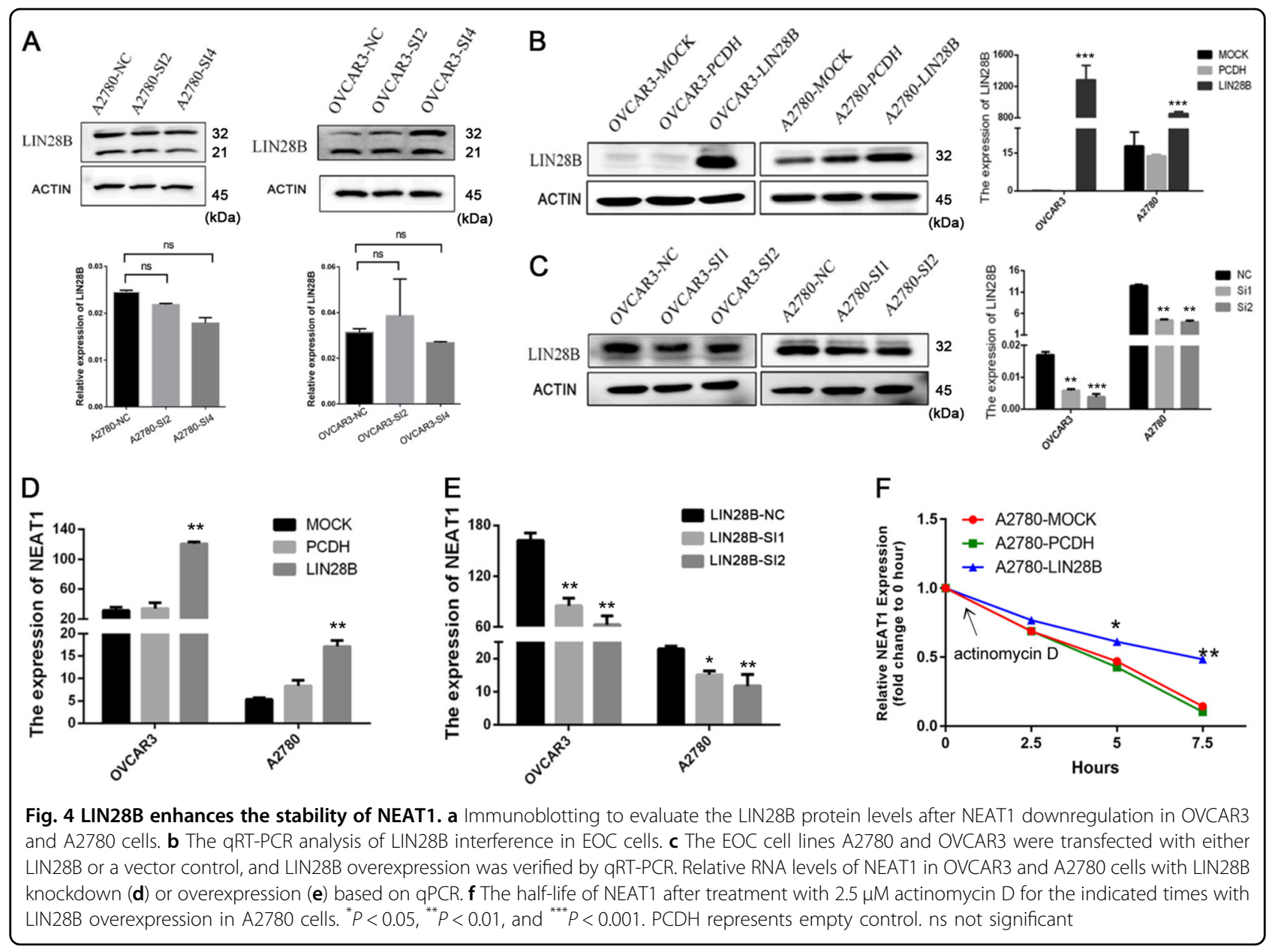

NEAT1 knockdown inhibits the growth of HGSOC xenografts in vivo

To provide in vivo evidence for the oncogenic role of NEAT1 in HGSOC, first, we constructed NEAT1 knockdown stable cell lines; the knockdown efficiency is shown in Fig. 6a. Then, we used a xenograft mouse model. After subcutaneous injection of 10 mice with A2780-NC and A2780-shRNA cells, all of the mice developed detectable tumors. The growth of the xenograft was significantly inhibited in mice treated with the NEAT1 shRNA lentivirus compared with that in mice treated with the NC lentivirus. (Fig. 6b-d). To investigate the functions of NEAT1 in vivo, we used frozen mouse tissue sections to conduct an immunofluorescence assay. The results showed that Ki67, which is a proliferation index, had brighter fluorescence in the NEAT1-NC group than in the NEAT1-shRNA group (Fig. 6e). LIN28B expression of mice tumor tissues was detected by Western blot and immunofluorescence assay. The results revealed no differences in immunoblotting strips and fluorescence intensity between the NEAT1-NC group and the NEAT1shRNA group (Supplementary Figure S3A, S3B), which provided strong support for the previous findings that NEAT1 had no effect on LIN28B.

\section{NEAT1 regulates tumor cell-cell adhesion via miR-506}

To identify the genes targeted by NEAT 1 and to explore the mechanism of NEAT1 in tumorigenesis, gene expression profiling was performed on NEAT1-siRNA HeLa and control cells (GSE45158) ${ }^{24}$. A total of 387 differentially expressed genes (DEGs; $>1$-fold; false discovery rate $=0.05$ ) were identified (Supplementary Table 2). A heat map of these DEGs is presented in Fig. 7a. Then, we conducted a gene annotation analysis through Metascape (http://metascape.org; Supplementary Table 3). The enrichment analysis showed that NEAT1 influenced multiple biological processes (Fig. 7b), including cell-cell adhesion, which plays pivotal roles in tumor cell proliferation and metastasis. We also selected a subset of representative terms from this cluster and converted them into a network layout (Fig. 7c).

Previous studies have shown that many lncRNAs function as ceRNAs for specific miRNAs ${ }^{25}$. To clarify the mechanism by which NEAT1 affects cell-cell 


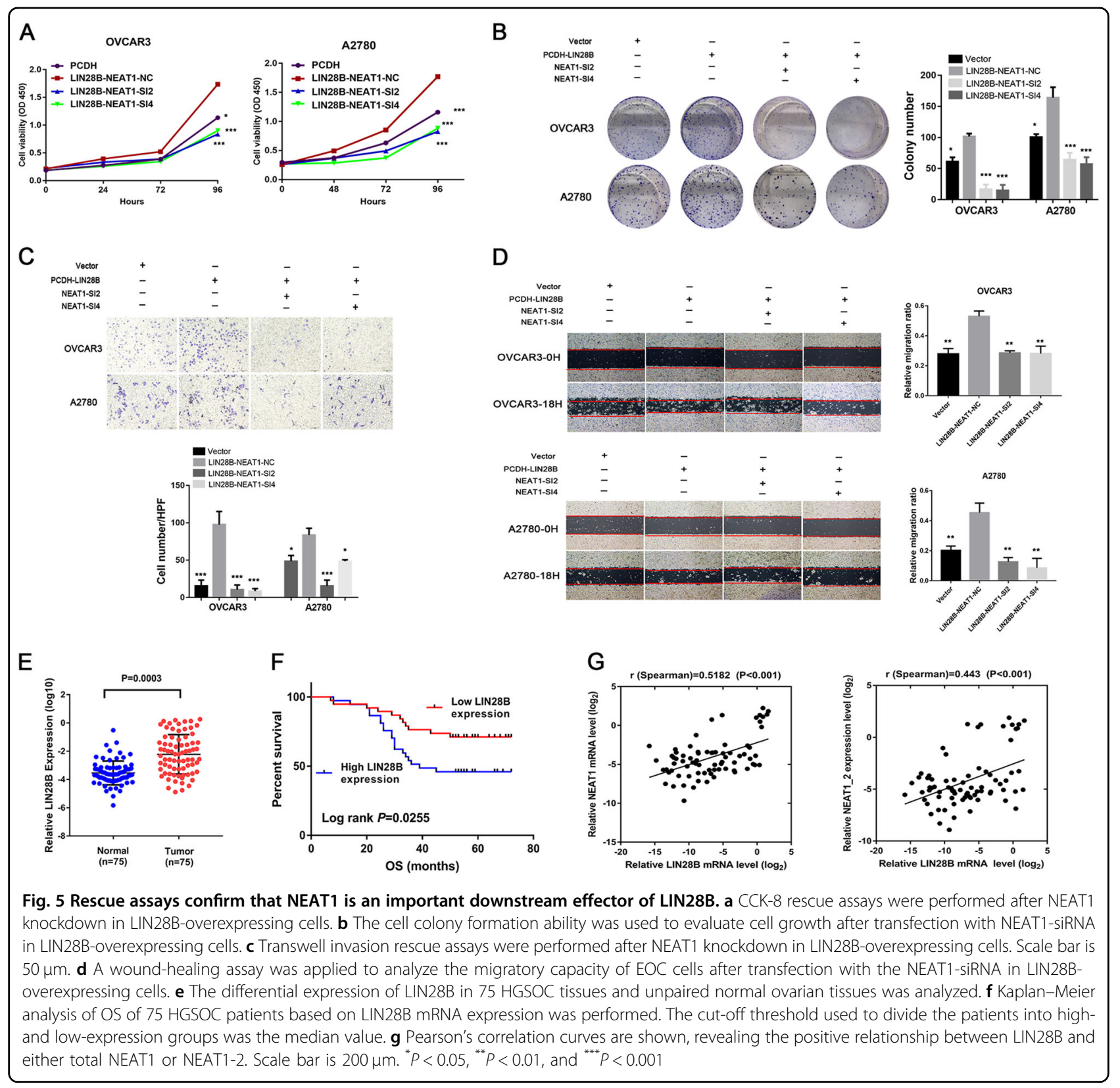

adhesion, we focused on whether NEAT1 could act as a ceRNA. Target prediction tools (ChipBase and StarBase) and a literature review were applied to evaluate potential miRNAs. Among these miRNAs, miR-506 was chosen as the predicted candidate because (1) the expression of miR-506 was upregulated after NEAT1 knockdown (Fig. 7d), (2) it contained NEAT1 binding sites, and (3) it has a strong effect on the EMT ${ }^{26,27}$. The complementary binding sites between miR-506 and NEAT1 are shown in Fig. 7e. To validate whether miR-506 was a direct target of NEAT1, luciferase reporter plasmids expressing NEAT1 with wild type/mutant miR-506 binding sites were constructed in our study (Fig. 7e). Cotransfection of HEK-293T cells with the luciferase reporter plasmid containing the wild type binding sites and miR506 mimics decreased the reporter activity relative to that in the negative control (Fig. 7f). Additionally, because miR-506 has been reported to target EMTrelated genes, such as ZEB1, Vimentin, and Snail2, we examined whether NEAT1/miR-506 could influence these proteins. Overexpression of miR-506 downregulated ZEB1, Vimentin, and Snail2 in A2780 cells (Fig. $7 \mathrm{~g}$ ), and consistent results were found when NEAT1 was knocked down by siRNAs (Fig. 7h). 


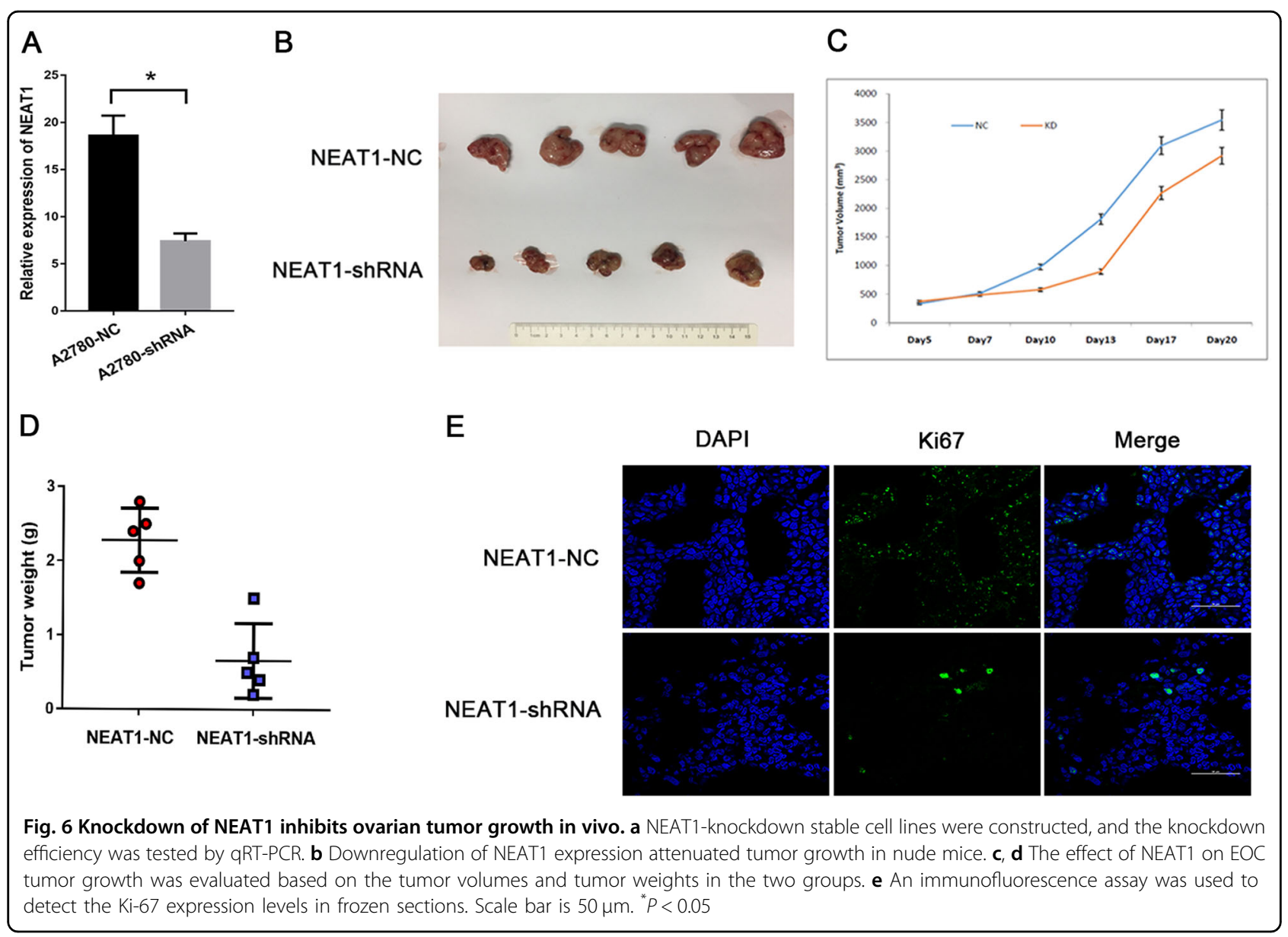

To further test our ceRNA hypothesis, rescue experiments were conducted. Knockdown of the endogenous highly expressed NEAT1 by NEAT1-SI2 significantly weakened the migration and invasion of OVCAR3 and A2780 cells (Fig. 8a, b). Suppression of miR-506 blocked the effects induced by NEAT1 depletion (Fig. 8a, b). Moreover, knocking down miR-506 alone greatly enhanced the invasive ability (Fig. 8a, b). The expression of the miR-506 targeted genes ZEB1, Vimentin, and Snail2 was analyzed. The influence of siNEAT1 on expression was reversed by miR-506 inhibitor in ovarian cancer cells (Fig. 8c). These results demonstrate that NEAT1 promotes tumor cell proliferation and metastasis according to the ceRNA patterns (Fig. 8d).

\section{Discussion}

With the recent development of high-throughput techniques, a variety of systematic cancer genomics projects have been conducted to investigate different molecular pathways and to identify genomic, transcriptomic, and epigenomic alterations in cancers ${ }^{28,29}$. These investigations have focused not only on protein-coding genes but also on long noncoding transcripts, which have been shown to be involved in the regulation of a diverse array of biological processes. This study focused on NEAT1, which is a lncRNA that has been studied as the core structural component of paraspeckles ${ }^{7,8,10}$. A great deal of effort has been expended to understand the functional role of NEAT1 in cancer progression. For example, Zhen et al. showed that NEAT1 promoted glioma pathogenesis by interacting with the miR-449b-5p/c-Met axis ${ }^{30}$. In addition, NEAT1 was found to promote papillary thyroid cancer through regulating ATAD2 expression by sponging miR-106b-5 $\mathrm{p}^{31}$. In regards to chemo-resistance, NEAT1 was found to upregulate EGCG-induced CTR1 and enhance cisplatin sensitivity in lung cancer cells ${ }^{32}$. Moreover, NEAT1 was found to be associated with malignant progression in ovarian cancer and was reported to have clinical significance ${ }^{17,33,34}$. However, elucidating its detailed involvement in ovarian tumorigenesis requires further investigation.

In our study, we found that the relative expression of NEAT1 was significantly increased in HGSOC tissues. High NEAT1 expression was correlated with tumor size. Additionally, NEAT1 was a potential independent prognostic factor for HGSOC. After knocking down NEAT1, 


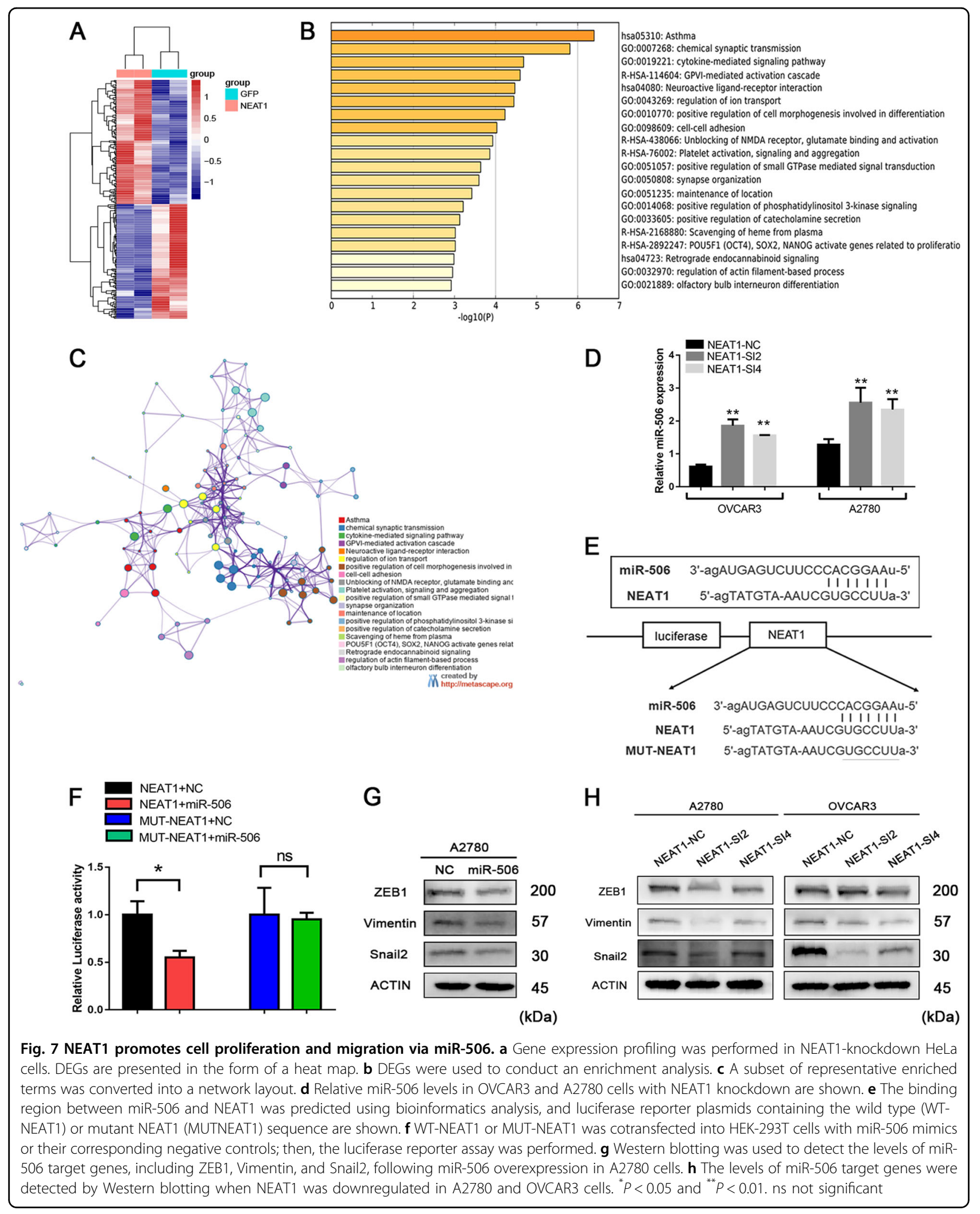




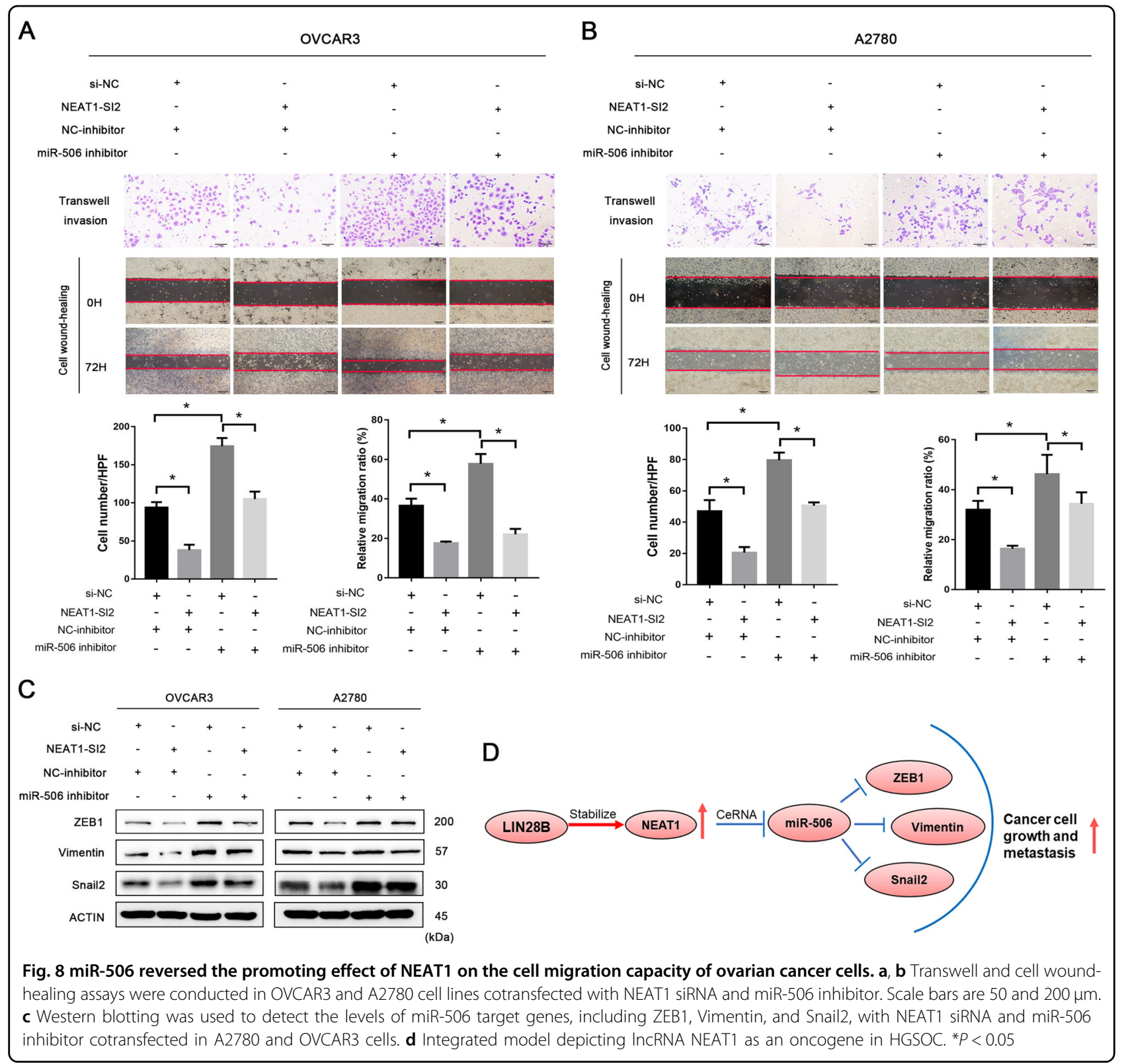

the malignant behaviors of ovarian cancer cells were restrained. These results are consistent with those of the previous studies and support a role for NEAT1 as a functional oncogenic IncRNA in HGSOC.

Next, we sought to identify the underlying molecular mechanisms by which NEAT1 exerts its modulatory effect on malignant EOC phenotypes. Previous studies reported that posttranscriptional regulation through specific RBPs was emerging as a critical regulatory level for nearly all biological processes ${ }^{19}$. Recent reports have shown that NEAT1 interacts with some RBPs. Jiang et al. found that NEAT1 bound the RBPs NONO and PSF to enhance premiRNA processing 22 . To examine whether NEAT1 could bind to other RBPs in ovarian cancer cells, we performed a bioinformatics analysis, RNA pulldown assay, and RIP assay, which revealed that NEAT1 could combine with LIN28B.

The molecular mechanisms underlying the function of LIN28B, as a member of the RBP family, were initially suggested to predominantly include regulation of the let-7 miRNAs, which have essential functions in inflammation, wound healing, embryonic stem cells, and cancer ${ }^{35-39}$. LIN28B expression is associated with tumor initiation, progression, resistance, and poor outcomes in several solid cancers ${ }^{40-43}$, including ovarian cancer ${ }^{44-46}$. However, few reports have investigated the interactions 
between LIN28B and lncRNAs. Only lncRNA H19 has been found to interact with LIN28B ${ }^{23}$. Our results extend current knowledge on the functional role of lncRNA-LIN28 complexes. However, the longer transcript of NEAT1 has a weak binding capacity with LIN28B. There are a few reasons why the longer transcript NEAT1_2 has a much weaker binding capacity with LIN28B than does NEAT1_1. First, the two transcripts have different lengths, which may result in different secondary structures. Second, it is believed that NEAT1_2, not NEAT1_1, is indispensable for paraspeckle formation. Although they both promote tumorigenesis, these two NEAT1 isoforms are reported to be involved in different cellular activities ${ }^{47}$, suggesting that the binding capacity with LIN28B may differ between the two transcripts. Third, recent reports have shown that NEAT1_2 interacts with some $\mathrm{RBPs}^{48}$. It is possible that other RBPs may affect the combination of LIN28B and NEAT1_2. Fourth, primers are responsible for different binding capacities. In the present study, we designed two primers named NEAT1 (which can detect both transcripts) and NEAT1-2 (which can only detect the long transcript), so the abundance of NEAT1 in cells differed between the two primers used for detection. The present study identified LIN28B as an important player in the regulation of NEAT1 expression by enhancing its stability. Rescue assays confirmed the function of the LIN28B/NEAT1 axis in ovarian cancer cells.

Additional findings of the present study involve the identification of downstream molecules associated with NEAT1 functions. The data obtained from the GEO database (GEO accession: GSE45158) indicated that NEAT1 influences multiple biological processes, including cell-cell adhesion. Cell adhesion plays a pivotal role in tumorigenesis and is regarded as an important component of the EMT. Emerging evidence suggests that lncRNAs may function as ceRNAs or molecular sponges to modulate the activities of miRNAs. To further elaborate the relationship between NEAT1 and EMT, we investigated whether NEAT1 could act as a ceRNA. As a recognized EMT-associated molecule, miR-506 has been reported to be expressed in many types of cancer and to regulate EMT-associated genes, such as Snail2, Vimentin, and ETS- $1^{27,49,50}$. Luciferase assays and Western blotting showed that NEAT1 might sponge miR-506 to promote tumorigenesis. Future work is needed to elucidate the detailed mechanism by which NEAT1 regulates miR-506 and to further delineate the network controlled by NEAT1 during HGSOC progression.

In summary, this study is the first report to investigate a potential interaction between NEAT1 and LIN28B during HGSOC progression. We found that NEAT1 was overexpressed and was associated with poor patient survival. NEAT1 promoted EOC cell proliferation, tumor invasion and metastasis in vitro, and cell proliferation in vivo. Moreover, we observed that NEAT1 might promote tumorigenesis by sponging miR-506. Our findings provide new insights into the molecular details of HGSOC and potential therapeutic targets to combat this disease.

\section{Materials and methods \\ Human samples and tissue handling}

The study was undertaken with the understanding and written consent of each subject. The study methodologies conformed to the standards set by the Declaration of Helsinki. All human tissues were collected using the protocols approved by the Human Ethics Committee of the Fudan University Shanghai Cancer Center. HGSOC tissues were obtained from 75 patients who underwent surgical resection of ovarian cancer between 2009 and 2012. Normal ovarian tissues were collected from cervical cancer surgery patients. No local or systemic treatment was administered to these patients prior to the operation. All tissue samples were washed with sterile phosphatebuffered saline before being snap frozen in liquid nitrogen and stored at $-80^{\circ} \mathrm{C}$ until analysis. The pathological parameters were appraised by an experienced pathologist. Patient follow-up was performed every 3 months during the first year postsurgery and then every 3-6 months thereafter until April 1, 2018. Disease-free survival (DFS) was calculated from the date of surgery to the date of recurrence or the last follow-up as appropriate.

\section{RNA isolation, reverse transcription, and quantitative real- time PCR}

Total RNA was extracted from the tissue samples and cell lines using TRIzol reagent (Invitrogen, Carlsbad, CA, USA) according to the manufacturer's protocol. Reverse transcription (RT) and qRT-PCR kits (Takara, Dalian, China) were utilized to evaluate the mRNA expression levels of the indicated genes. PCR primers were designed and synthesized using a primer design tool (http://www. ncbi.nlm.nih.gov/tools/primer-blast/); the primer sequences are listed in Supplementary Table 4. The relative quantification value for each target gene was expressed as $2^{-\Delta \Delta C T}$. $\beta$-Actin was used as an internal reference for the mRNAs, and U6 served as an internal reference for the miRNAs.

\section{Cell culture and treatments}

HEK-293T cells and the human EOC cell lines A2780, HO8910, SKOV3, OVCAR3, CAOV3, ES-2, and OV420 were cultured in Dulbecco's modified Eagle's medium (Gibco, Carlsbad, CA, USA) supplemented with 10\% fetal bovine serum (FBS) (Gibco), $50 \mathrm{U} / \mathrm{mL}$ of penicillin and 50 $\mu \mathrm{g} / \mathrm{mL}$ of streptomycin (Gibco). All cell lines were maintained at $37^{\circ} \mathrm{C}$ and $5 \% \mathrm{CO}_{2}$ in a humidified atmosphere. 
Lentivirus, siRNA, miR-506 inhibitor, and transfection

The NEAT1-shRNA-Lentivirus for use in the in vivo experiments was purchased from the ABM Company. LIN28B-related stable cell lines were constructed according to previously published guidelines ${ }^{51}$. siRNAs targeting NEAT1 and LIN28B were purchased from Suzhou Synbio Technologies and transfected into cells using Lipofectamine 3000 (Invitrogen). All siRNA and shRNA sequences are provided in Supplementary Table 5. miR-506 inhibitor was purchased from GenePharma (Shanghai, China).

\section{Cell proliferation assays}

Cells $\left(2 \times 10^{3}\right.$ per well $)$ were seeded into 96 -well plates $24 \mathrm{~h}$ before the experiment. A2780 and OVCAR3 cells were transfected with siRNAs or a scramble siRNA. Proliferation was measured using the CCK-8 kit (Dojindo, Japan) according to the manufacturer's protocol. All experiments were performed in triplicate. The cell proliferation curves were plotted using the absorbance at each time point.

\section{Colony formation assay}

Cells were resuspended into single-cell suspensions $48 \mathrm{~h}$ posttransfection. For the colony formation assay, 1000 cells were plated into 6-well plates and incubated in the corresponding medium with $10 \%$ FBS at $37^{\circ} \mathrm{C}$. After 2 weeks, the cells were fixed and stained with $0.1 \%$ crystal violet. The visible colonies were manually counted. Triplicate wells were measured for each treatment group.

\section{Cell wound-healing and invasion assays}

For the wound-healing assay, cells were seeded into 6well plates and allowed to grow to $90-95 \%$ confluence. A single scratch wound was created $6 \mathrm{~h}$ after transfection with the siRNAs. The cells were washed with PBS to remove cell debris, supplemented with serum-free medium, and monitored. Images were captured by phasecontrast microscopy at $0,18,24$, and $36 \mathrm{~h}$ after wounding.

A cell invasion assay was performed using Transwell chamber inserts $(8.0 \mathrm{~mm}$, Corning, NY, USA) in a 24 -well plate. Then, $2 \times 10^{4}$ cells suspended in $200 \mu \mathrm{L}$ of serumfree medium were added to the upper chamber. Culture medium containing 20\% FBS was placed in the bottom chamber. The cells were incubated for 24 or $48 \mathrm{~h}$ at $37^{\circ} \mathrm{C}$. After incubation, the cells on the upper surface were scraped and washed away, whereas the cells on the lower surface were fixed with $20 \%$ methanol and stained with $0.1 \%$ crystal violet. The numbers of invaded cells were counted in five randomly selected fields under a microscope. The experiments were repeated independently in triplicate.

\section{RNA pulldown assays}

Due to the long length of NEAT1, we designed two probes (wild type and mutant) that we labeled using the Biotin RNA Labeling Mix (Thermo Fisher, USA), treated with RNase-free DNase I (Takara, Japan) and purified with the RNeasy Mini Kit (QIAGEN, USA). Next, 1 pmol of biotinylated RNA was pretreated with RNA structure buffer (Beyotime Biotechnology, Shanghai, China) to ensure appropriate secondary structure formation. The pretreated biotinylated RNAs were incubated with $1 \mathrm{mg}$ of protein extract from the A2780 cells at $4{ }^{\circ} \mathrm{C}$ for $4 \mathrm{~h}$, gently mixed with $40 \mu \mathrm{L}$ of washed streptavidin beads (Invitrogen, CA, USA) and incubated on a rotator overnight according to the manufacturer's protocol (Thermo Fisher, Waltham, USA). The proteins were precipitated and diluted in $60 \mu \mathrm{L}$ of protein lysis buffer and then detected by Western blotting.

\section{RBP immunoprecipitation and RIP-qPCR assays}

We performed RIP experiments using a Magna RIP RNA-Binding Protein Immunoprecipitation Kit (Millipore, Billerica, MA, USA) according to the manufacturer's instructions. A rabbit anti-LIN28B antibody (1:50; Cell Signaling Technology) was used for the experiments. The lysates were incubated with the antibody overnight at $4{ }^{\circ} \mathrm{C}$. The coprecipitated RNAs were detected by real-time PCR. The corresponding primers are provided in Supplementary Table 4.

\section{Western blotting assay}

Cells were lysed in RIPA buffer (Sigma-Aldrich) supplemented with a protease inhibitor (Roche, Basel, Switzerland) and a phosphatase inhibitor (Roche). The protein concentration was measured using a BCA protein assay kit (Thermo Scientific, USA). The primary rabbit antiLIN28B, anti-MMP2, anti-MMP9, anti-N-Cadherin, and anti-E-Cadherin antibodies were purchased from Cell Signaling Technology. The rabbit anti-ZEB1, antivimentin, and anti-Snail2 antibodies were purchased from Proteintech.

\section{Mouse xenografts and immunofluorescence}

Female BALB/c nude mice 4-5 weeks of age were purchased from the Shanghai Laboratory Animal Center at the Chinese Academy of Sciences. All experiments were performed in accordance with relevant institutional and national guidelines and the regulations of the Shanghai Medical Experimental Animal Care Commission. Mice (5 per group) were injected subcutaneously with $0.2 \mathrm{~mL}$ of a cell suspension containing $5 \times 10^{5}$ cells (the piLentishRNA-VECTOR and piLenti-shRNA-NEAT1 stable A2780 cell lines) in the right axilla. The tumor growth rates were monitored. When a tumor was palpable, it was 
measured every other day, and its volume was calculated according to the formula: volume $=$ length $\times$ width $^{2} \times 0.5$.

To investigate the relationship between NEAT1 and LIN28B in vivo, frozen sections from animal experimental tumors were washed with PBS and fixed with $4 \%$ paraformaldehyde for $20 \mathrm{~min}$ at room temperature. After three washes with PBS, the sections were blocked in $5 \%$ goat serum for $1 \mathrm{~h}$. The cells were subsequently incubated with primary antibodies specific for Ki-67 (1:100, Proteintech) and LIN28B (1:50, Abcam; ab71415) overnight at $4{ }^{\circ} \mathrm{C}$. The next day, the sections were washed with PBS and then incubated with fluorescence-conjugated secondary antibodies (Beyotime, China), followed by DAPI. Images were captured under a fluorescence microscope.

\section{Luciferase assay}

The wild type (WT) or mutant (MUT) NEAT1-binding sites were subcloned into the pGL3 Basic vector (Promega, Madison, WI, USA). HEK-293T cells were seeded onto 96-well plates. Mimics of miR-506 or an NC (RiboBio, Guangzhou, China) were cotransfected with pLUC-WT-NEAT1 or pLUC-MUT-NEAT1. Two days after transfection, the cells were collected, and the luciferase activity was determined using the Dual Luciferase Reporter Assay System (Promega, Madison, WI, USA).

\section{GEO dataset analysis}

The GSE45158 dataset acquired from the GEO database was analyzed using GEO2R (https://www.ncbi.nlm.nih. gov/geo/geo2r/) to identify DEGs following NEAT1 downregulation. Genes with $P$ values $<0.05$ and $|\operatorname{logFC}|>$ 1 were considered DEGs. In total, 387 DEGs were identified. Then, gene annotation analysis was conducted with Metascape (http://metascape.org).

\section{Statistical analysis}

All statistical analyses were performed using SPSS 18.0 (IBM, SPSS, Chicago, IL, USA). The significance of differences between groups was estimated using Student's $t$ test, the $\chi^{2}$ test, or the Wilcoxon test as appropriate. The OS and DFS rates were calculated using the Kaplan-Meier method with the log-rank test for comparison. The survival data were evaluated using univariate and multivariate Cox regression analyses. A value of $P<$ 0.05 indicated a significant difference.

\section{Acknowledgements}

This work was supported in part by grants from the National Natural Science Foundation of China (No. 81672569) and the Science and Technology Commission of Shanghai Municipality (15411962000 and 17411963000).

\footnotetext{
Author details

'Department of Gynecologic Oncology, Fudan University Shanghai Cancer Center, Shanghai, China. ${ }^{2}$ Department of Oncology, Shanghai Medical College, Fudan University, Shanghai, China. ${ }^{3}$ Department of Pathology, Fudan University Shanghai Cancer Center, Shanghai, China
}

Conflict of interest

The authors declare that they have no conflict of interest.

\section{Publisher's note}

Springer Nature remains neutral with regard to jurisdictional claims in published maps and institutional affiliations.

Supplementary Information accompanies this paper at (https://doi.org/ 10.1038/s41419-018-0908-z).

Received: 10 May 2018 Revised: 6 July 2018 Accepted: 22 July 2018 Published online: 28 August 2018

\section{References}

1. Barnett, R. Ovarian cancer. Lancet 387, 1265 (2016).

2. Crum, C. P. Preventing ovarian cancer. J. Clin. Oncol. 34, 198-199 (2016).

3. Bertone-Johnson, E. R. Epidemiology of ovarian cancer: a status report. Lancet 365, 101-102 (2005).

4. Chen, Y. G., Satpathy, A. T. \& Chang, H. Y. Gene regulation in the immune system by long noncoding RNAs. Nat. Immunol. 18, 962-972 (2017).

5. $\mathrm{Xu}, \mathrm{Q}$. et al. Long non-coding RNA regulation of epithelial-mesenchymal transition in cancer metastasis. Cell Death Dis. 7, e2254 (2016).

6. Wahlestedt, C. Targeting long non-coding RNA to therapeutically upregulate gene expression. Nat. Rev. Drug Discov. 12, 433-446 (2013).

7. Imamura, K. et al. Long noncoding RNA NEAT1-dependent SFPQ relocation from promoter region to paraspeckle mediates IL8 expression upon immune stimuli. Mol. Cell 53, 393-406 (2014).

8. Yu, X., Li, Z., Zheng, H., Chan, M. T. \& Wu, W. K. NEAT1: a novel cancer-related long non-coding RNA. Cell Prolif. 50, https://doi.org/10.1111/cpr.12329 (2017).

9. Adriaens, C. et al. p53 induces formation of NEAT1 IncRNA-containing paraspeckles that modulate replication stress response and chemosensitivity. Nat. Med. 22, 861-868 (2016).

10. Shevtsov, S. P. \& Dundr, M. Nucleation of nuclear bodies by RNA. Nat. Cell Biol. 13, 167-173 (2011).

11. Wu, F., Mo, Q., Wan, X., Dan, J. \& Hu, H. NEAT1/has-mir-98-5p/MAPK6 axis is involved in non-small-cell lung cancer (NSCLC) development. J. Cell Biochem. https://doi.org/10.1002/jcb.26442 (2017).

12. Zheng, X. et al. HIF-2alpha activated IncRNA NEAT1 promotes hepatocellular carcinoma cell invasion and metastasis by affecting the epithelial-mesenchymal transition. J. Cell. Biochem. 119, 3247-3256 (2018).

13. Xiong, W. et al. Oncogenic non-coding RNA NEAT1 promotes the prostate cancer cell growth through the SRC3/IGFIR/AKT pathway. Int. J. Biochem. Cell Biol. 94, 125-132 (2018).

14. $\mathrm{Wu}, \mathrm{Y}$. et al. Nuclear-enriched abundant transcript 1 as a diagnostic and prognostic biomarker in colorectal cancer. Mol. Cancer 14, 191 (2015).

15. Liu, F., Tai, Y. \& Ma, J. LncRNA NEAT1/let-7a-5p axis regulates the cisplatin resistance in nasopharyngeal carcinoma by targeting Rsf-1 and modulating the Ras-MAPK pathway. Cancer Biol. Ther. 19, 534-542 (2018).

16. Cheng, N. \& Guo, Y. Long noncoding RNA NEAT1 promotes nasopharyngeal carcinoma progression through regulation of miR-124/NF-kappaB pathway. OncoTargets Ther. 10, 5843-5853 (2017).

17. Chai, Y., Liu, J., Zhang, Z. \& Liu, L. HuR-regulated IncRNA NEAT1 stability in tumorigenesis and progression of ovarian cancer. Cancer Med. 5, 1588-1598 (2016).

18. Jens, M. \& Rajewsky, N. Competition between target sites of regulators shapes post-transcriptional gene regulation. Nat. Rev. Genet. 16, 113-126 (2015).

19. Chatterji, P. \& Rustgi, A. K. RNA binding proteins in intestinal epithelial biology and colorectal cancer. Trends Mol. Med. 24(5), 490-506 (2018).

20. Militti, C., Maenner, S., Becker, P. B. \& Gebauer, F. UNR facilitates the interaction of MLE with the InCRNA roX2 during Drosophila dosage compensation. Nat. Commun. 5, 4762 (2014).

21. Ferre, F., Colantoni, A. \& Helmer-Citterich, M. Revealing protein-IncRNA interaction. Brief. Bioinformatics 17, 106-116 (2016).

22. Jiang, L. et al. NEAT1 scaffolds RNA-binding proteins and the microprocessor to globally enhance pri-miRNA processing. Nat. Struct. Mol. Biol. 24, 816-824 (2017). 
23. Helsmoortel, H. H. et al. LIN28B is over-expressed in specific subtypes of pediatric leukemia and regulates IncRNA H19. Haematologica. 101, e240-e244 (2016).

24. Hirose, T. et al. NEAT1 long noncoding RNA regulates transcription via protein sequestration within subnuclear bodies. Mol. Biol. Cell 25, 169-183 (2014).

25. Thomson, D. W. \& Dinger, M. E. Endogenous microRNA sponges: evidence and controversy. Nat. Rev. Genet. 17, 272-283 (2016).

26. Seviour, E. G. et al. Functional proteomics identifies miRNAs to target a p27/ $\mathrm{Myc} /$ phospho- $\mathrm{Rb}$ signature in breast and ovarian cancer. Oncogene $\mathbf{3 5}$, 691-701 (2016).

27. Sun, Y. et al. MiR-506 inhibits multiple targets in the epithelial-to-mesenchymal transition network and is associated with good prognosis in epithelial ovarian cancer. J. Pathol. 235, 25-36 (2015).

28. Kandoth, C. et al. Mutational landscape and significance across 12 major cancer types. Nature 502, 333-339 (2013)

29. Alvarez, M. J. et al. Functional characterization of somatic mutations in cancer using network-based inference of protein activity. Nat. Genet. 48, 838-847 (2016).

30. Zhen, L., Yun-Hui, L., Hong-Yu, D., Jun, M. \& Yi-Long, Y. Long noncoding RNA NEAT1 promotes glioma pathogenesis by regulating miR-449b-5p/c-Met axis. Tumour Biol. 37, 673-683 (2016).

31. Sun, W. et al. NEAT1_2 functions as a competing endogenous RNA to regulate ATAD2 expression by sponging microRNA-106b-5p in papillary thyroid cancer. Cell Death Dis. 9, 380 (2018).

32. Jiang, P., Wu, X., Wang, X., Huang, W. \& Feng, Q. NEAT1 upregulates EGCGinduced CTR1 to enhance cisplatin sensitivity in lung cancer cells. Oncotarget 7, 43337-43351 (2016)

33. An, J., Lv, W. \& Zhang, Y. LncRNA NEAT1 contributes to paclitaxel resistance of ovarian cancer cells by regulating ZEB1 expression via miR-194. OncoTargets Ther. 10, 5377-5390 (2017).

34. Ding, N., Wu, H., Tao, T. \& Peng, E. NEAT1 regulates cell proliferation and apoptosis of ovarian cancer by miR-34a-5p/BCL2. OncoTargets Ther. 10, 4905-4915 (2017).

35. Graf, R. et al. Identification of LIN28B-bound mRNAs reveals features of target recognition and regulation. RNA Biol. 10, 1146-1159 (2013).

36. Shyh-Chang, N. \& Daley, G. Q. Lin28: primal regulator of growth and metabolism in stem cells. Cell Stem Cell 12, 395-406 (2013).
37. Thornton, J. E. \& Gregory, R. I. How does Lin28 let-7 control development and disease?. Trends Cell Biol. 22, 474-482 (2012).

38. Jiang, S. \& Baltimore, D. RNA-binding protein Lin28 in cancer and immunity. Cancer Lett. 375, 108-113 (2016).

39. Wang, T. et al. Aberrant regulation of the LIN28A/LIN28B and let-7 loop in human malignant tumors and its effects on the hallmarks of cancer. Mol. Cancer 14, 125 (2015).

40. Meder, L. et al. LIN28B enhanced tumorigenesis in an autochthonous KRAS (G12V)-driven lung carcinoma mouse model. Oncogene. https:/doi.org/ 10.1038/s41388-018-0158-7 (2018).

41. Powers, J. T. et al. Multiple mechanisms disrupt the let-7 microRNA family in neuroblastoma. Nature 535, 246-251 (2016).

42. Molenaar, J. J. et al. LIN28B induces neuroblastoma and enhances MYCN levels via let-7 suppression. Nat. Genet. 44, 1199-1206 (2012).

43. Nguyen, L. H. et al. Lin28b is sufficient to drive liver cancer and necessary for its maintenance in murine models. Cancer Cell 26, 248-261 (2014).

44. Hsu, K. F. et al. Overexpression of the RNA-binding proteins Lin28B and IGF2BP3 (IMP3) is associated with chemoresistance and poor disease outcome in ovarian cancer. Br. J. Cancer 113, 414-424 (2015).

45. Lu, L. et al. LIN-28B/let-7a/IGF-II axis molecular subtypes are associated with epithelial ovarian cancer prognosis. Gynecol. Oncol. 141, 121-127 (2016).

46. Lu, L. et al. Functional study of risk loci of stem cell-associated gene lin-28B and associations with disease survival outcomes in epithelial ovarian cancer. Carcinogenesis 33, 2119-2125 (2012).

47. Li, W. et al. The FOXN3-NEAT1-SIN3A repressor complex promotes progression of hormonally responsive breast cancer. J. Clin. Invest. 127, 3421-3440 (2017).

48. Yamazaki, T. et al. Functional domains of NEAT1 architectural IncRNA induce paraspeckle assembly through phase separation. Mol. Cell 70, 1038-1053 (2018).

49. Li, Z. et al. miR-506 inhibits epithelial-to-mesenchymal transition and angiogenesis in gastric cancer. Am. J. Pathol. 185, 2412-2420 (2015).

50. Li, D. et al. miR-506 suppresses neuroblastoma metastasis by targeting ROCK1. Oncol. Lett. 13, 417-422 (2017).

51. Weng, W. et al. PTTG3P promotes gastric tumour cell proliferation and invasion and is an indicator of poor prognosis. J. Cell Mol. Med. 21, 3360-3371 (2017). 